\title{
Stability Analysis of Newly Evolved Genotypes of Chrysanthemum (Dendranthema grandiflora Tzvelev) for Cut Flower Production using Eberhart and Russel Model
}

\author{
Reshma Negi ${ }^{1 *}$, R. K. Dogra ${ }^{1}$, S. R. Dhiman ${ }^{1}$, Y. C. Gupta ${ }^{1}$, \\ R. K. Gupta ${ }^{1}$ and M. R. Dhiman ${ }^{2}$ \\ ${ }^{1}$ College of Horticulture, Dr. Yashwant Singh Parmar University of Horticulture \\ and Forestry, Nauni, Solan, Himachal Pradesh-173230, India \\ ${ }^{2}$ ICAR-IARI Regional Research Station, Katrain, Kullu, India \\ *Corresponding author
}

A B S T R A C T

K e y w o r d s
$\begin{aligned} & \text { Chrysanthemum, } \\ & \text { Stability, Cut } \\ & \text { flower, phenotypic } \\ & \text { index, Regression } \\ & \text { Coefficient }\end{aligned}$
Article Info
$\begin{aligned} & \text { Accepted: } \\ & 15 \text { May } 2020 \\ & \text { Available Online: } \\ & \text { 10 June } 2020\end{aligned}$

Study was carried out to determine the stability of genotypes of chrysanthemum (Dendranthema grandiflora Tzvelev) for cut flower production at the experimental farm of Department of Floriculture and Landscape Architecture, Dr. Yashwant Singh Parmar University of Horticulture and Forestry, Nauni, Solan and ICAR-IARI Regional Research Station, Katrain, Kullu Valley of H.P for two successive years 2017 and 2018 on nineteen genotypes of chrysanthemum, including 'Ajay' as check using Eberthart and Russel model. The character wise stable genotypes were ranked based on their stability for different characters 'UHFSChr132' recorded stable performance for days taken to flowering, plant spread, flower size 'UHFSChr122' for number of cut stems and weight of cut stems. Genotype 'UHFSChr125' for days taken to flowering, stem length.

\section{Introduction}

Chrysanthemum (Dendranthema grandiflora Tzvelev) is a multi use flower crop, belongs to family Asteraceae. It is native to northern hemisphere chiefly Europe and Asia. It is national flower of Japan and species in the genus chrysanthemum varies from 100 to 200 . It ranks second after rose (Anonymous,
2017). In India during 2016-2017 the area under chrysanthemum was 20090 hectare and production of cut flower was 14930 MT (Anonymous, 2018). Total area under chrysanthemum in Himachal Pradesh for cut flower is $67.01 \mathrm{ha}$ and with annual production of 5360 lakh number of cut flower respectively during the year 2018-2019 (Anonymous, 2019). 
It is most popular due to its wide range of flower colour, growth habit, size and shape. It is used for interior decoration and in bouquets. The tall growing plants are suitable for back ground planting in borders. The utility and popularity of chrysanthemum have increased immensely with the introduction of the techniques for year round blooming based on scientific research in the field of photoperiodism and genetics.

However, ability to produce chrysanthemum year round depends on an understanding the complex interaction between the plant and its environment. A sound knowledge of its physiology and management practices can ensure the continued success of chrysanthemum crop despite increasing production cost.

Somehow, the available cultivars could not fulfill the requirements in terms of new colors, forms, types and various characteristics. However, very little attention has been paid for its improvement. Therefore, there is urgent need to for identification of varieties suitable for growing in different agro climatic conditions for specific purposes. Hence, it is utmost important to develop such genotypes which are stable over wide range of environment conditions.

Therefore, an investigation was undertaken on "Stability analysis in chrysanthemum (Dendranthema grandiflora Tzvelev) as 'stability' reflects the suitability of genotype for general cultivation over wide range of environment for cut flower production".

According to the dynamic concept, a stable genotype is one which gives predictable performance to environments without any deviation (Becker and Leon, 1988). Identification of stable genotype will help the farmers for its successful commercial cultivation.

\section{Materials and Methods}

An experiment was conducted on stability analysis of newly evolved genotypes of chrysanthemum for cut flower production at experimental farm of Department of Floriculture and Landscape Architecture, Dr. Yashwant Singh Parmar University of Horticulture and Forestry, Nauni, Solan and ICAR-IARI, Regional Research Station, Katrain, Kullu Valley of H.P for two successive years 2017 and 2018 on nineteen genotypes of chrysanthemum.

Genotype namely 'UHFSChr111', 'UHFSChr113', 'UHFSChr114', 'UHFSChr115', 'UHFSChr117', 'UHFSChr118', 'UHFSChr120', 'UHFSChr121', 'UHFSChr122', 'UHFSChr123', 'UHFSChr124', 'UHFSChr125', 'UHFSChr126', 'UHFSChr128', 'UHFSChr129', 'UHFSChr130', 'UHFSChr131', 'UHFSChr132' including 'Ajay' as check.

The plants were planted in three replications in Randomized Block Design in open field conditions using FYM $5 \mathrm{~kg} / \mathrm{m}^{2}$ and half dose of nitrogen and full dose of phosphorus and potassium was also mixed in the soil at the time of bed preparation.

The remaining half dose of nitrogen was applied 45 days after transplanting. Data were recorded in terms of different plant parameters viz., days taken for flowering, plant height $(\mathrm{cm})$ recorded at the time of flowering and measured from bottom to tip of the plant, stem length, stem weight, flower diameter $(\mathrm{cm})$ and duration of flowering.

The data was subjected to stability analysis by using Eberhart and Russell (1966) stability model. The model involves the estimation of mean, regression coefficient and deviation from regression. 


\section{Results and Discussion}

Pooled analysis of data on stability analysis of nineteen genotypes of chrysanthemum for two years at two locations are presented in Table 1, Table 2, Table 3, Table 4, Table 5, and Table 6.

Data presented in Table 1 revealed that there were significant difference among genotypes for stability analysis with respect to plant height shows that genotypes 'UHFSChr114' $(114.97 \mathrm{~cm}), \quad$ 'UHFSChr115' (114.90cm), 'UHFSChr117' (116.08cm), 'UHFSChr125' (90.33cm), 'UHFSChr131'(87.00cm), 'UHFSC $\mathrm{hr} 132$ ' $(88.33 \mathrm{~cm})$ recorded significantly high mean values than overall mean $(86.78 \mathrm{~cm})$. From among above mentioned genotypes 'UHFSChr115' was found to have phenotypic index (pi) 5.95, regression coefficient (bi) 1.40 and squared deviation from regression $\left(\mathrm{S}^{2} \mathrm{di}\right) 0.08$ and this genotype found stable for plant height.

Days taken to flower bud formation genotypes 'UHFSChr129' (135.35 days), 'UHFSChr120' (126.13 days), 'UHFSChr 114' (125.88 days), 'UHFSChr124' (125.08 days), 'UHFSChr130' (125.04 days) 'UHFSChr126' (124.73days), 'UHFSChr113' (124.60 days), 'UHFSChr125' (124.47 cm), exhibited significantly more mean values than overall mean (124.40 days).

From above mentioned genotypes 'UHFSChr126' observed value of phenotypic index (pi) 96.54, regression coefficient (bi) 0.99 and squared deviation from regression $\left(S^{2} \mathrm{di}\right)$ 0.96. Hence, this genotype was found stable for trait under study.

Days taken to flowering the genotypes 'UHFSChr129' (163.15 days), 'UHFSChr 120' (161.82 days), 'UHFSChr126' (161.23 days), 'UHFSChr113' (160.85 days), ('UHFSChr111' (160.75 days), 'Ajay' (160.47 days), UHFSChr132’ (160.23 days),
UHFSChr 125' (160.23days),UHFSChr124', (159.50days), 'UHFSChr114' (158.93 days) and 'UHFSChr115' (158.75cm) showed significantly high mean values than overall mean (158.23 days).The genotypes 'UHFSChr115', 'UHFSChr125' and 'UHFSChr132'recorded phenotypic index (pi) 99.43, 96.23 and 117.10 regression coefficient (bi) value 0.99 each and squared deviation from regression $\left(\mathrm{S}^{2} \mathrm{di}\right) 2.79,15.50$ and 4.62 respectively revealed that these genotypes as rich environment.

Significant variation were noted for plant spread among genotypes across the environments and genotypes 'UHFSChr121' $(38.29 \mathrm{~cm}), \quad$ 'UHFSChr114' (36.36 cm), 'UHFSChr122' (35.60 cm), 'UHFSChr117' $(35.29 \mathrm{~cm})$, 'UHFSChr128' (35.28 cm), 'UHFSChr120' (34.74 cm), 'UHFSChr125' (34.77 cm), 'UHFSChr130' (34.52 cm), 'UHFSChr132' (34.41 cm) recorded high mean values than the overall mean (34.37 days). The above mentioned genotypes did not fulfill the criteria to be stable, hence they were found to be unstable for plant spread.

The duration of flowering exhibited significant variations among genotypes 'UHFSChr126'(35.42 days), 'UHFSChr132' (35.33 days), 'UHFSChr111' (35.00days), 'Ajay' (34.75 days), 'UHFSChr130' (34.67 days), 'UHFSChr124' (33.67 days), and 'UHFSChr120' (33.25 days), exhibited significantly high mean values than the overall mean (29.22 days).

Among above said genotypes 'UHFSChr132' revealed phenotypic index (pi) 1.18, regression coefficient (bi) 1.72 and 0.03 squared deviation from regression $\left(\mathrm{S}^{2} \mathrm{di}\right)$ was found to be most stable for the trait under study.

A perusal of data presented in Table 3 revealed that there were significant variations in flower diameter. The genotypes 
UHFShr124'(10.19cm), 'UHFSChr115' (6.33cm), 'UHFSChr113' $(6.05 \mathrm{~cm})$, ,UHFSChr $123^{\prime}(5.49 \mathrm{~cm}),{ }^{\prime} \mathrm{UHFSChr} 129$ '(5.38cm), 'UHF SChr120' $(5.27 \mathrm{~cm}),{ }^{\prime} \mathrm{UHFSChr} 126^{\prime}(5.21 \mathrm{~cm}),{ }^{\circ}$ UHFSChr111'(5.20cm), 'UHFSChr130'(5.14 $\mathrm{cm})$ showed maximum diameter of flowers that overall mean $(5.05 \mathrm{~cm})$.Genotypes 'UHFSChr132' observed phenotypic index (pi) 1.18, regression coefficient (bi) 1.93 and squared deviation from regression $\left(\mathrm{S}^{2} \mathrm{di}\right)$ 0.03 . Hence this genotypes was found to be the most stable for flower size.

Data presented on stability analysis for stem length noted significant variations (Table 4 ) observed among genotypes 'UHFSChr115'(97.00 cm), 'UHFSChr114'(78.84 cm), 'UHFSChr125' $(78.50 \mathrm{~cm}), \quad$ 'UHFSChr117'(77.28 cm), 'UHFSChr122'(75.25 cm), 'UHFSChr121' $(73.83 \mathrm{~cm}), \quad$ 'UHFSChr132' $(73.08 \mathrm{~cm})$ 'UHFSChr123'(71.33 cm) recorded high values for stem length than overall mean value $(71.28 \mathrm{~cm})$.Among above specified genotypes 'UHFSChr123' and 'UHFSChr125'exhibited high phenotypic index (pi), regression coefficient (bi) 1.01, 1.65 and squared deviations from regression ( $\left.\mathrm{S}^{2} \mathrm{di}\right) 9.73$ and 4.70 respectively. It indicated that these two genotypes were suitable to rich environment.

For weight of cut stem (g) the genotypes 'UHFSChr111'(43.00 gm), 'UHFSChr126' (42.08gm), 'Ajay'(39.08gm)'UHFSChr132'(3 8.83gm), 'UHFSChr122'(38.42gm), 'UHFSCh r130'(38.25 gm), 'UHFSChr124'(32.25 gm) and 'UHFSChr128' (32.33 gm) recorded significant high mean values than the overall mean (31.93 gm).From above said genotypes 'UHFSChr122' and 'UHFSChr128' observed phenotypic index (pi) 12.27, 10.13, regression coefficient (bi) 1.32, 1.45 and squared deviation from regression $\left(\mathrm{S}^{2}\right.$ di) $-0.91,-0.05$ showing that these genotypes were suitable for average environments.

Data for number of flowers per stem showed that genotypes 'UHFSChr128' (73.27), 'UHFSChr132' (71.22), 'UHFSChr117' (69.88), 'UHFSChr114' (56.25) 'UHFSChr122' (54.78), 'UHFSChr121' (47.55), 'UHFSChr113' (46.70), 'UHFSChr111' (46.25), and 'UHFSChr 131'(44.91) and 'UHFSChr125' recorded maximum mean values than the overall mean (40.63).

Among above mentioned genotypes 'UHFSChr121' recorded phenotypic index (pi) 11.34, high regression coefficient (bi) 1.98 and squared deviation from regression $\left(\mathrm{S}^{2} \mathrm{di}\right)-6.90$ was found stable with average environment.

The present study revealed that the genotypes 'UHFSChr132', 'UHFSChr122' and 'UHFSChr125' observed stable for days taken to flowering, duration of flowering and flower size. Genotype 'UHFSChr122' for number of stems per plant, weight of cut stem and genotype 'UHFSChr 125' days taken to flowering and stem length for cut flower.

These genotypes were less influenced by the season as well as environment and hence found to be stable across the locations and seasons. Similar results have been observed in different floricultural crops; chrysanthemum by Vaidya (2006), Priyanka (2012) and Kumar et al., (2018); gladiolus; Ramberg and Chirva (1978), Arora and Sharma (1991), Desh Raj and Misra (1998 a, b); carnation by Pant and Lal (1998), Misra et al., (2002); marigold by, Naik (2003), Patil et al., (2011) in line with the present findings. 
Table.1 Estimation of stability parameters in chrysanthemum for plant height $(\mathrm{cm})$, days taken to flower bud formation

\begin{tabular}{|c|c|c|c|c|c|c|c|c|}
\hline \multirow[t]{2}{*}{ Genotype } & \multirow[b]{2}{*}{ Mean } & \multicolumn{3}{|c|}{ Plant height $(\mathbf{c m})$} & \multirow[b]{2}{*}{ Mean } & \multicolumn{3}{|c|}{$\begin{array}{l}\text { Days taken to flower bud } \\
\text { formation }\end{array}$} \\
\hline & & pi & $\mathbf{B i}$ & $S^{2} d i$ & & $\mathbf{P i}$ & bi & $S^{2} d i$ \\
\hline UHFSChr 111 & 68.15 & -25.88 & 1.72 & 10.06 & 122.87 & 73.04 & 0.99 & 5.76 \\
\hline UHFSChr 113 & 83.67 & 12.79 & 1.54 & -1.56 & 124.60 & 330.40 & 0.94 & 3.52 \\
\hline UHFSChr 114 & 114.97 & -25.04 & 0.29 & 7.32 & 125.88 & -3299.01 & 1.11 & 13.21 \\
\hline UHFSChr 115 & 114.90 & 5.95 & 1.40 & 0.08 & 124.27 & -111.73 & 1.02 & 1.12 \\
\hline UHFSChr 117 & 116.08 & 15.68 & 0.78 & -0.36 & 124.29 & 9.84 & 1.00 & -0.57 \\
\hline UHFSChr 118 & 75.53 & 19.57 & 1.77 & -0.55 & 124.00 & 97.52 & 0.99 & -0.60 \\
\hline UHFSChr 120 & 71.33 & 18.21 & 0.16 & -0.79 & 126.13 & -66.56 & 1.02 & -0.23 \\
\hline UHFSChr 121 & 84.68 & 14.67 & -0.01 & 1.08 & 122.70 & 177.91 & 0.97 & 0.74 \\
\hline UHFSChr 122 & 82.31 & 8.52 & 1.50 & -0.46 & 123.98 & 371.52 & 0.93 & 4.97 \\
\hline UHFSChr 123 & 82.53 & 20.41 & 0.89 & -0.62 & 119.55 & -942.99 & 1.15 & 43.44 \\
\hline UHFSChr 124 & 70.42 & 20.12 & 0.56 & -0.64 & 125.08 & 30.77 & 1.00 & 9.16 \\
\hline UHFSChr 125 & 90.33 & 10.12 & 0.17 & 1.87 & 124.47 & 70.15 & 0.99 & -1.13 \\
\hline UHFSChr 126 & 82.75 & -20.99 & 1.25 & 8.47 & 124.73 & 96.54 & 0.99 & 0.96 \\
\hline UHFSChr 128 & 84.68 & 13.48 & 2.47 & 0.01 & 124.07 & 82.40 & 0.99 & -0.94 \\
\hline UHFSChr 129 & 85.08 & -25.57 & -1.07 & 14.35 & 130.35 & 807.16 & 0.84 & 5.59 \\
\hline UHFSChr 130 & 85.27 & -0.84 & 0.88 & 7.02 & 125.04 & 181.28 & 0.97 & 1.86 \\
\hline UHFSChr 131 & 87.00 & -22.24 & 1.65 & 9.10 & 123.63 & -52.22 & 1.01 & 0.77 \\
\hline UHFSChr 132 & 88.33 & -68.99 & 1.54 & 26.98 & 124.12 & -22.29 & 1.01 & 2.05 \\
\hline Ajay & 80.85 & 7.59 & 1.52 & 16.35 & 123.78 & -533.93 & 1.10 & 5.33 \\
\hline Pooled Mean & 86.78 & & $\begin{array}{r}\text { SE( } \\
\text { SE( } \\
\text { Mean }\end{array}$ & $\begin{array}{l}=1.56 \\
0.91 \\
b=1.00\end{array}$ & 124.40 & & $\begin{array}{r}\text { SE } \\
\text { SE } \\
\text { Mea }\end{array}$ & $\begin{array}{l}=1.51 \\
0.05 \\
b=1.00\end{array}$ \\
\hline
\end{tabular}


Table.2 Estimation of stability parameters in chrysanthemum for days taken to flowering and plant spread

\begin{tabular}{|c|c|c|c|c|c|c|c|c|}
\hline \multirow[t]{2}{*}{ Genotype } & \multirow[b]{2}{*}{ Mean } & \multicolumn{3}{|c|}{ Days taken to flowering } & \multirow[b]{2}{*}{ Mean } & \multicolumn{3}{|c|}{ Plant spread (cm) } \\
\hline & & pi & bi & $S^{2} \mathrm{di}$ & & pi & bi & $\mathrm{S}^{2} \mathrm{di}$ \\
\hline UHFSChr 111 & 160.75 & 445.87 & 0.88 & 20.39 & 34.94 & 15.83 & 0.49 & -1.53 \\
\hline UHFSChr 113 & 160.85 & -49.20 & 1.02 & 28.23 & 33.87 & 6.32 & 1.86 & 2.97 \\
\hline UHFSChr 114 & 158.93 & -2463.48 & 1.17 & 9.78 & 36.36 & -5.28 & -4.02 & -0.30 \\
\hline UHFSChr 115 & 158.75 & 99.43 & 0.99 & 2.79 & 33.86 & 11.45 & 4.13 & -0.68 \\
\hline UHFSChr 117 & 149.68 & 300.29 & 0.81 & 192.62 & 35.29 & -21.43 & 14.04 & 1.33 \\
\hline UHFSChr 118 & 158.57 & 213.92 & 0.96 & -0.98 & 33.94 & -2.30 & 8.63 & 1.59 \\
\hline UHFSChr 120 & 161.82 & -257.25 & 1.09 & 1.48 & 34.74 & -17.64 & 4.32 & 13.74 \\
\hline UHFSChr 121 & 155.35 & -156.67 & 1.06 & 5.30 & 38.39 & -46.16 & -14.29 & 13.13 \\
\hline UHFSChr 122 & 153.20 & -276.84 & 1.10 & -1.53 & 35.60 & 10.91 & -3.08 & 0.20 \\
\hline UHFSChr 123 & 156.75 & -386.80 & 1.12 & 10.18 & 33.43 & 12.68 & 3.33 & -0.82 \\
\hline UHFSChr 124 & 159.50 & -346.82 & 1.09 & 44.48 & 26.75 & -27.53 & -9.03 & 13.65 \\
\hline UHFSChr 125 & 160.23 & 96.23 & 0.99 & 15.50 & 34.77 & 1.36 & -1.78 & 5.48 \\
\hline UHFSChr 126 & 161.23 & 204.50 & 0.95 & 23.99 & 34.98 & 11.34 & -3.15 & -0.05 \\
\hline UHFSChr 128 & 158.95 & 129.08 & 0.99 & -1.23 & 35.28 & 13.99 & 0.55 & -0.61 \\
\hline UHFSChr 129 & 163.15 & 209.37 & 0.96 & 7.69 & 33.58 & 7.08 & 6.10 & -0.11 \\
\hline UHFSChr 130 & 156.09 & -48.45 & 1.03 & 6.86 & 34.52 & 10.96 & 2.91 & 0.25 \\
\hline UHFSChr 131 & 151.92 & 442.32 & 0.75 & 209.41 & 34.33 & 10.56 & -5.61 & -1.39 \\
\hline UHFSChr 132 & 160.23 & 117.10 & 0.99 & 4.62 & 34.41 & -6.14 & 5.03 & 7.45 \\
\hline Ajay & 160.47 & -129.46 & 1.05 & 13.95 & 34.06 & -2.09 & 8.56 & 1.59 \\
\hline Pooled Mean & 158.23 & & $\begin{array}{r}\text { SE } \\
\text { SE } \\
\text { Mean }\end{array}$ & $\begin{array}{l}=3.31 \\
0.13 \\
p=1.00\end{array}$ & 34.37 & & $\begin{array}{r}\text { S E } \\
\text { S E } \\
\text { Mean }\end{array}$ & $\begin{array}{l}1.24 \\
5.35 \\
=1.00\end{array}$ \\
\hline
\end{tabular}


Table.3 Estimation of stability parameters in chrysanthemum for duration of flowering (days) and flower diameter $(\mathrm{cm})$

\begin{tabular}{|c|c|c|c|c|c|c|c|c|}
\hline \multirow[t]{2}{*}{ Genotype } & \multirow[b]{2}{*}{ Mean } & \multicolumn{3}{|c|}{$\begin{array}{c}\text { Duration of flowering } \\
\text { (days) }\end{array}$} & \multirow[b]{2}{*}{ Mean } & \multicolumn{3}{|c|}{ Flower diameter $(\mathrm{cm})$} \\
\hline & & pi & bi & $S^{2} d i$ & & pi & bi & $S^{2} d i$ \\
\hline UHFSChr 111 & 35.00 & 3.18 & -0.10 & -0.62 & 5.20 & 0.76 & -1.62 & -0.02 \\
\hline UHFSChr 113 & 25.92 & -0.90 & 3.99 & -0.48 & 6.05 & -0.07 & 2.06 & 0.28 \\
\hline UHFSChr 114 & 25.08 & -1.64 & -0.06 & -0.25 & 4.03 & -0.09 & 0.47 & -0.01 \\
\hline UHFSChr 115 & 25.92 & 3.32 & 1.05 & -0.82 & 6.33 & -1.56 & 3.67 & 0.38 \\
\hline UHFSChr 117 & 27.00 & 3.85 & -0.54 & -0.99 & 3.59 & 1.03 & 0.88 & -0.02 \\
\hline UHFSChr 118 & 25.67 & 3.85 & 0.81 & -1.03 & 3.90 & 0.43 & -0.81 & 0.28 \\
\hline UHFSChr 120 & 33.25 & -30.24 & 9.33 & 5.74 & 5.27 & 1.04 & 0.14 & 0.02 \\
\hline UHFSChr 121 & 24.50 & -0.04 & 2.34 & 0.34 & 4.15 & 0.69 & 1.59 & 0.02 \\
\hline UHFSChr 122 & 27.50 & 3.51 & 0.84 & -0.87 & 4.98 & -0.32 & 0.47 & 0.69 \\
\hline UHFSChr 123 & 26.67 & 3.85 & -0.32 & -0.97 & 5.49 & -8.24 & 5.76 & 2.33 \\
\hline UHFSChr 124 & 33.67 & -6.82 & -5.78 & 0.41 & 10.19 & 1.11 & 0.07 & -0.01 \\
\hline UHFSChr 125 & 24.75 & 3.76 & -0.28 & -0.92 & 4.29 & 0.21 & 2.30 & 0.07 \\
\hline UHFSChr 126 & 35.42 & 3.32 & 0.04 & -0.69 & 5.21 & -0.10 & 0.49 & 0.58 \\
\hline UHFSChr 128 & 25.83 & 2.18 & -0.61 & -0.17 & 3.63 & 1.00 & -0.75 & 0.01 \\
\hline UHFSChr 129 & 26.17 & 0.85 & 3.27 & -0.73 & 5.38 & 0.42 & 1.82 & 0.10 \\
\hline UHFSChr 130 & 34.67 & -1.04 & 4.52 & -0.94 & 5.14 & 1.10 & -0.44 & -0.02 \\
\hline UHFSChr 131 & 28.08 & 0.65 & -3.13 & -0.53 & 4.06 & 0.54 & -0.67 & 0.24 \\
\hline UHFSChr 132 & 35.33 & 1.18 & 1.72 & 0.03 & 4.97 & 0.90 & 1.05 & 0.02 \\
\hline Ajay & 34.75 & 3.10 & 1.93 & -1.02 & 4.03 & -0.01 & 2.51 & 0.10 \\
\hline Pooled Mean & 29.22 & & $\begin{array}{l}\text { SE(r } \\
\text { SE(b } \\
\text { Mean }\end{array}$ & $\begin{array}{l}0.53 \\
1.86 \\
=1.00\end{array}$ & & 5.05 & $\begin{array}{r}\text { SE } \\
\text { SE } \\
\text { Mear }\end{array}$ & $\begin{array}{l}=0.32 \\
1.46 \\
=1.00\end{array}$ \\
\hline
\end{tabular}


Table.4 Estimation of stability parameters in chrysanthemum for stem length $(\mathrm{cm})$ and number of cut stems per plant

\begin{tabular}{|c|c|c|c|c|c|c|c|c|}
\hline \multirow[t]{2}{*}{ Genotype } & \multirow[b]{2}{*}{ Mean } & \multicolumn{3}{|c|}{ Stem length $(\mathrm{cm})$} & \multirow[b]{2}{*}{ Mean } & \multicolumn{3}{|c|}{$\begin{array}{l}\text { Number of cut stems per } \\
\text { plant }\end{array}$} \\
\hline & & pi & bi & $S^{2} d i$ & & $\mathbf{P i}$ & bi & $\mathbf{S}^{2} \mathrm{di}$ \\
\hline UHFSChr 111 & 56.33 & 339691.27 & -0.82 & -0.61 & 4.75 & 0.45 & -0.90 & 0.03 \\
\hline UHFSChr 113 & 63.58 & 339827.79 & -1.44 & 2.54 & 6.17 & 0.42 & -0.25 & 0.09 \\
\hline UHFSChr 114 & 78.84 & -6453927.63 & 6.99 & 79.18 & 6.58 & -0.53 & 0.52 & 0.06 \\
\hline UHFSChr 115 & 97.00 & 338814.02 & -1.40 & 15.50 & 6.00 & -1.24 & 4.00 & -0.07 \\
\hline UHFSChr 117 & 77.27 & 339794.77 & 1.27 & -1.25 & 6.50 & 0.87 & -0.29 & -0.14 \\
\hline UHFSChr 118 & 65.17 & 339637.46 & 4.37 & 1.12 & 4.48 & 0.45 & 0.03 & 0.08 \\
\hline UHFSChr 120 & 64.58 & 339084.68 & 1.72 & 11.97 & 5.53 & 0.81 & 1.13 & -0.18 \\
\hline UHFSChr 121 & 73.83 & 339839.91 & -0.70 & 0.45 & 5.42 & -1.10 & 3.48 & 0.10 \\
\hline UHFSChr 122 & 75.25 & 339741.46 & 2.80 & 18.81 & 6.67 & 0.76 & 1.12 & -0.15 \\
\hline UHFSChr 123 & 71.33 & 339839.49 & 1.01 & 9.73 & 5.18 & 0.84 & 0.44 & -0.13 \\
\hline UHFSChr 124 & 58.58 & 339614.16 & -0.68 & 26.25 & 3.92 & -2.88 & -0.85 & 1.70 \\
\hline UHFSChr 125 & 78.50 & 339778.57 & 1.65 & 4.70 & 6.00 & -0.13 & -2.17 & 0.08 \\
\hline UHFSChr 126 & 70.67 & 339807.49 & 0.32 & 21.56 & 5.70 & 0.39 & 0.21 & 0.11 \\
\hline UHFSChr 128 & 70.92 & 339830.60 & -0.88 & 7.29 & 5.60 & -0.50 & 3.25 & -0.11 \\
\hline UHFSChr 129 & 71.00 & 339840.60 & -0.24 & -0.72 & 4.92 & -0.88 & 3.85 & -0.18 \\
\hline UHFSChr 130 & 71.08 & 339815.02 & -1.52 & 5.88 & 5.12 & 0.03 & 2.42 & -0.07 \\
\hline UHFSChr 131 & 70.17 & 339839.04 & 0.23 & 0.79 & 5.67 & 0.09 & 2.33 & -0.08 \\
\hline UHFSChr 132 & 73.08 & 339782.91 & 3.36 & 26.20 & 6.75 & 0.67 & 0.77 & -0.07 \\
\hline Ajay & 67.08 & 339494.60 & 2.97 & 4.37 & 4.18 & 0.50 & -0.09 & 0.06 \\
\hline Pooled Mean & 71.28 & & $\begin{array}{r}\mathrm{SE}(1 \\
\mathrm{SE}(\mathrm{b} \\
\mathrm{M} \\
\mathrm{b}=\end{array}$ & $\begin{array}{l}=2.24 \\
=2.24 \\
\text { an of } \\
1.00\end{array}$ & 40.63 & 5.35 & $\begin{array}{r}\text { SE } \\
\text { SE( } \\
\text { Mean }\end{array}$ & $\begin{array}{l}=0.29 \\
1.39 \\
b=1.00\end{array}$ \\
\hline
\end{tabular}


Table.5 Estimation of stability parameters in chrysanthemum for weight of cut stem $(\mathrm{g})$ and number of flowers per stem

\begin{tabular}{|c|c|c|c|c|c|c|c|c|}
\hline \multirow[t]{2}{*}{ Genotype } & \multirow[b]{2}{*}{ Mean } & \multicolumn{3}{|c|}{ Weight of cut stem (g) } & \multicolumn{4}{|c|}{ Number of flowers per stem } \\
\hline & & pi & bi & $S^{2}$ di & Mean & $\mathbf{P i}$ & bi & $S^{2} \mathrm{di}$ \\
\hline UHFSChr 111 & 43.00 & 14.57 & -0.25 & -1.09 & 46.25 & 49.69 & -0.46 & 3.95 \\
\hline UHFSChr 113 & 27.83 & 5.80 & -1.74 & 1.56 & 46.70 & 50.54 & 0.74 & 0.83 \\
\hline UHFSChr 114 & 24.17 & -3.00 & 0.00 & -0.33 & 56.25 & -212.07 & -0.17 & 98.31 \\
\hline UHFSChr 115 & 25.00 & 5.24 & 0.80 & 3.24 & 32.67 & 53.93 & 1.05 & -5.40 \\
\hline UHFSChr 117 & 30.67 & -55.20 & -6.65 & 8.08 & 69.88 & 6.16 & 0.90 & 20.87 \\
\hline UHFSChr 118 & 26.83 & 14.24 & -0.50 & -1.04 & 29.81 & 50.13 & 0.38 & 4.30 \\
\hline UHFSChr 120 & 27.58 & 2.49 & 0.75 & 4.66 & 32.10 & 20.30 & 1.23 & 8.20 \\
\hline UHFSChr 121 & 26.50 & 14.24 & 0.55 & -1.06 & 47.55 & 11.34 & 1.98 & -6.90 \\
\hline UHFSChr 122 & 38.42 & 12.27 & 1.32 & -0.91 & 54.78 & -207.79 & 3.90 & 10.71 \\
\hline UHFSChr 123 & 25.50 & 4.91 & 2.19 & 1.00 & 11.99 & 68.02 & 0.46 & -5.24 \\
\hline UHFSChr 124 & 33.25 & 9.82 & 2.01 & -1.02 & 9.20 & 44.56 & 1.12 & -1.87 \\
\hline UHFSChr 125 & 29.00 & 10.57 & 0.54 & 0.78 & 41.19 & 14.90 & -0.27 & 22.47 \\
\hline UHFSChr 126 & 42.08 & -59.73 & 7.87 & -0.03 & 39.68 & 44.15 & 0.75 & 3.84 \\
\hline UHFSChr 128 & 32.33 & 10.13 & 1.45 & -0.05 & 73.27 & -118.52 & 2.01 & 57.11 \\
\hline UHFSChr 129 & 30.83 & -6.20 & 4.24 & -1.13 & 21.58 & 32.83 & 1.23 & 1.92 \\
\hline UHFSChr 130 & 38.25 & -10.84 & 3.80 & 3.23 & 19.99 & 51.06 & 1.13 & -5.37 \\
\hline UHFSChr 131 & 27.42 & 12.49 & -0.14 & -0.02 & 44.91 & -1.78 & 2.17 & -6.67 \\
\hline UHFSChr 132 & 38.83 & 4.02 & -0.03 & 4.22 & 71.22 & -74.12 & 0.25 & 67.09 \\
\hline Ajay & 39.08 & -1.95 & 2.80 & 2.64 & 22.97 & 40.64 & 0.62 & 7.04 \\
\hline Pooled Mean & 31.93 & & $\begin{array}{r}\mathrm{SE}( \\
\mathrm{SE}(\mathrm{l} \\
\mathrm{M} \\
\mathrm{b}=\end{array}$ & $\begin{array}{l}=1.00 \\
=1.61 \\
\mathrm{n} \text { of } \\
.00\end{array}$ & 40.63 & & $\begin{array}{c}\text { SE } \\
\text { SE } \\
\text { Mean }\end{array}$ & $\begin{array}{l}=2.71 \\
1.16 \\
o=1.00\end{array}$ \\
\hline
\end{tabular}

Table.6 Character wise stable performance of chrysanthemum genotype

\begin{tabular}{|c|l|l|}
\hline Sr No. & \multicolumn{1}{|c|}{ Characters } & \multicolumn{1}{c|}{ Cut flower } \\
\hline $\mathbf{1 .}$ & Plant height $(\mathrm{cm})$ & UHFSChr115 \\
\hline $\mathbf{2 .}$ & Days taken to flower bud formation & UHFSChr126 \\
\hline $\mathbf{3 .}$ & Days taken to flowering & $\begin{array}{l}\text { UHFSChr115, UHFSChr125, } \\
\text { UHFSChr132 }\end{array}$ \\
\hline $\mathbf{4 .}$ & Plant spread $(\mathrm{cm})$ & - \\
\hline $\mathbf{5 .}$ & Duration of flowering (days) & UHFSChr132 \\
\hline $\mathbf{6 .}$ & Flower size $(\mathrm{cm})$ & UHFSChr132 \\
\hline $\mathbf{7 .}$ & Stem length $(\mathrm{cm})$ & UHFSChr123,UHFSChr125 \\
\hline $\mathbf{8 .}$ & Number of cut stems /shoots per plant & UHFSChr120, UHFSChr122 \\
\hline $\mathbf{9 .}$ & Weight of cut stem $(\mathrm{g})$ & UHFSChr122, UHFSChr128 \\
\hline $\mathbf{1 0 .}$ & Number of flowers per stem & UHFSChr121 \\
\hline & & \\
\hline
\end{tabular}


The character wise stable genotypes and ranking of genotypes based on their stability for different characters are given in Table 6 . For cut flower genotypes 'UHFSChr132' recorded stable performance for days taken to flowering, plant spread, flower size 'UHFSChr122' for number of cut stems and weight of cut stems. Genotype 'UHFSChr125' for days taken to flowering, stem length.

\section{References}

Anderson RL.1987.Reclassification of genus chrysanthemum HortScience 22: 313.

Anonymous. 2017. Flora Holland https://www.royalfloraholland.com/en/s peciale-paginas/ in... /bloemenagenda2017.

Eberhart SA and Russell WA. 1966. Stability parameters for comparing varieties. Crop Science 6: 36-40.

Anonymous. 2017. Royal Floraholland Facts and Figures. https:// www.royalfloraholland.com.

Anonymous. 2018. Ministry of Agriculture, Government of India.(https:www.indiastat.com/).

Anonymous. 2019. Status of floriculture in Himachal Pradesh. Directorate of Horticulture, Shimla, Himachal Pradesh, India.

Becker and Leon,1988. Stability analysis in plant breeding. Plant Breeding, 101:123.

Desh Raj A and Misra RL. 1998a. Stability analysis in gladiolus I. Vegetative characters. Annals of Agriculture
Research, 19(4): 423-428.

Desh Raj and Misra RL. 1998 b. Stability analysis in gladiolus II. Floral characters. Journal of Ornamental Horticulture 1(2): 61-65.

Kumar CM, Dorajeerao PAVD, Susheela T. and Salma Z. 2018. Stability analysis for flowering characters of chrysanthemum genotypes. Plant Archives 18(2):2609-2616.

Machin BJ, Scopes NEA. 1978. Chrysanthemum year round growing. Blandford Press, Dorset UK. p 233.

Mishra S, Gupta YC, and AR Rao. 2003. Correlation and path analysis studies in carnation. Journal of Ornamental Horticulture 6 (1):24-28.

Naik BH. 2003. Stability analysis and standardization of production technology for flower and xanthophylls yield in marigold (Tagetes spp). Ph.D Thesis, Department of Horticulture, College of Agriculture, Dharwad, University of Agricultural Sciences, Dharwad. India 329.

Priyanka BM. 2012. Genotypic stability analysis in chrysanthemum (Dendranthema grandiflora Tzevlev). M.Sc Thesis, Department of Floriculture and Landscape Architecture, Kittur Rani Channama College of Horticulture, University of Horticultural Sciences, Bagalkot.124.

Tamberg TG and Chirva ZF. 1978. Effect of climatic conditions on gladiolus growth and development. Genetike Selektsii 62(3): 115-121.

\section{How to cite this article:}

Reshma Negi, R. K. Dogra, S. R. Dhiman, Y. C. Gupta, R. K. Gupta and Dhiman. M. R. 2020. Stability Analysis of Newly Evolved Genotypes of Chrysanthemum (Dendranthema grandiflora Tzvelev) for Cut Flower Production using Eberhart and Russel Model. Int.J.Curr.Microbiol.App.Sci. 9(06): 477-486. doi: https://doi.org/10.20546/ijcmas.2020.906.062 